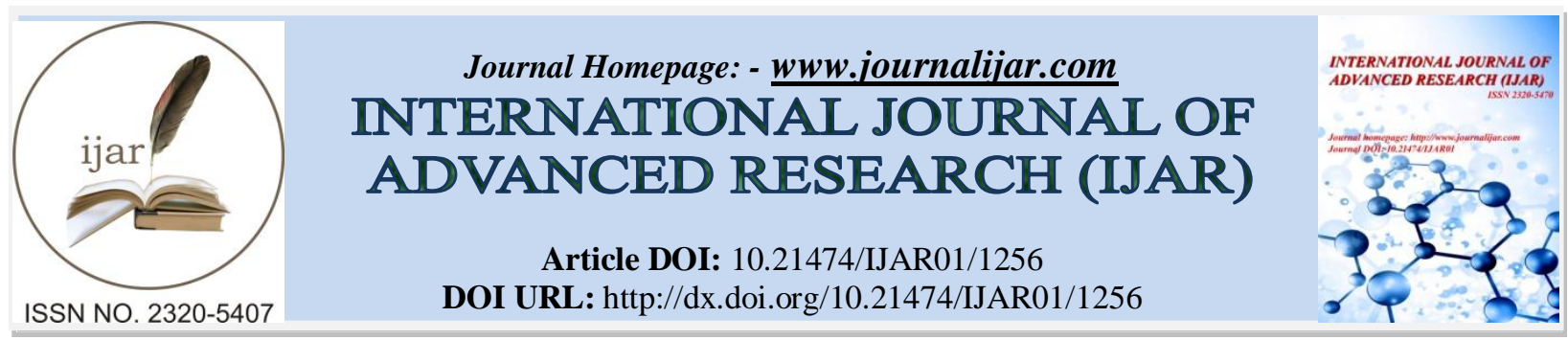

RESEARCH ARTICLE

\title{
Association of recombination errors and young mother age in risk of Down Syndrome in South Indian population.
}

\section{RaviRaj V Suresh ${ }^{1}$, Dr. Kusuma Lingaiah ${ }^{2}$, Dr. Narayannapa ${ }^{3}$ and Dr Savitha ${ }^{4}$ and Prof. Nallur B. Ramachandra ${ }^{1}$.}

1. Genetics and Genomics Lab, Department of Studies in Genetics and Genomics, University of Mysore, Manasagangotri, Mysuru-06.

2. Central Sericultural Research \& Training Institute, Manadawadi road, Srirampura, Mysuru-08, Karnataka, India.

3. Pediatrics Department, JSS Medical College, JSS University Mysuru-12.

4. Pediatrics Department Cheluvamba hospital, Mysore Medical College and Research institute Mysuru-01.

\section{Manuscript Info}

Manuscript History

Received: 16 June 2016

Final Accepted: 11 July 2016

Published: August 2016

Key words:-

nondisjunction; recombination; meiosis; insights; maternal age.

\begin{abstract}
Down syndrome (DS) is caused due to nondisjunction (NDJ) of chromosome 21; $90 \%$ of the cases are of maternal origin and is of major public health concern. Maternal age and altered recombination are the two well established risk factors causing NDJ. In the present study we have examined the association between these two factors using short tandem repeat (STR) markers in order to give further insights into the mechanism of NDJ. We have created maternal age stratified recombination profile which includes the recombination frequency and recombination location in 145 cases of mothers with DS and control mothers. Our investigation identifies parental origin, stage of NDJ and recombination pattern. The result from the study indicates Meiosis I NDJ errors are associated with altered recombination and young mother age. Recombination in telomeric region and multiple crossover events marked a significant impact on younger women with DS in the present investigation. In conclusion, our examination reveals recombination error, recombination pattern and maternal age play a major role in chromosomal NDJ and can act as predisposing factors for NDJ of chromosome 21 leading to DS.
\end{abstract}

Copy Right, IJAR, 2016,. All rights reserved.

\section{Introduction:-}

Down Syndrome (DS) (MIM 190685) is a type of mental retardation caused by extra genetic material of chromosome 21. This is due to a process called nondisjunction (NDJ), resulting in trisomy 21. The estimated incidence of DS is between 1 in 1,000 to 1 in 1,100 live births worldwide (WHO, 2015). In the majority of these cases the errors occur during maternal oogenesis (Antonarakis, 1991; Freeman et al., 2007), predominantly at meiosis I (MI) (Antonarakis, 1992; Sherman et al., 2007). Oogenesis is a lengthy process and involves meiotic arrest, which makes it more susceptible to malsegregation of chromosomes than spermatogenesis (Oliver et al., 2008). 
Advanced maternal age, advanced maternal grandmother age and altered recombination (Yoon et.al, 1996, Malini et.al, 2006, Oliver et al., 2008, Gosh et.al, 2009) are the major established risk factors for DS. Advances in mother age will bring swift degradation of cellular proteins involved in spindle formation, sister chromatid cohesion or anaphase separation of sister chromatids in oocytes (Hawley et al., 1994, Wolstenholme and Angell, 2000), which force the risk of nondisjunction (NDJ) both at MI and MII (Yoon et al., 1996).

Recombination, an important process in the fetal ovary, as it stabilizes the tetrad and helps in proper separation of chromatids to opposite poles. This is a random process and it may be absent even in euplod populations (Cheung et al, 2007). It has been shown that nondisjoined chromosomes often show altered patterns of recombination and location (MacDonald et al., 1994; Hassold et al., 1995; Koehler et al, 1996b). For trisomy 21, with reference to recombination errors, researchers have previously observed that single telomeric exchange as risk factor for NDJ regardless of maternal age (Lamb et.al, 2005, Oliver et.al, 2008, Ghosh et.al, 2010, Vranekovic et.al, 2012). These investigations were reported in different ethnic backgrounds and conclude the role of recombination errors in NDJ causing DS. Hence, here we present a similar study on recombination errors to verify its role in NDJ in south Indian ethnicity. Our present investigation reports the recombination profile in association with maternal age in South Indian population and is of particular importance as the population is very diverse and has higher inbreeding frequency.

\section{Materials and methods:-}

The present investigation includes 145 families with DS kids and 130 families with normal kids. Ethical clearance was obtained from Institutional Human Ethical Committee (IHEC), University of Mysore. All the Subjects for the study were recruited from Cheluvamba hospital and J.S.S Hospital, Pediatrics Department, Mysore and all participants were recruited after informed written consent. The study was conducted for a period of three years from 2012-2015.

\section{Criteria for sample collection:-}

Clinical data from the pediatricians and assessment of characteristic features of DS helped in enrolling the DS mothers. Kids of the enrolled DS mothers were subjected for Karyotyping. Only those mothers of the kids with confirmed free trisomy of chromosome 21 by karyotype analysis were selected for the study.

\section{Blood Sample Collection and Genomic DNA isolation:-}

$5 \mathrm{ml}$ of peripheral venous blood was collected in EDTA vacutainer from the DS families which include father, mother and their progeny with DS. Genomic DNA was isolated using Promega Wizard ${ }^{\circledR}$ Genomic DNA purification kit and quantified using spectrophotometer to know the concentration of the individual sample. All the DNA samples were used for further downstream PCR process.

\section{Detection of stage of meiotic nondisjunction and crossover events:-}

The obtained samples were genotyped by STR markers specific to chromosome 21 (Figure 1). Three pericentromeric markers D21S369, D21S215 and D21S258, were used to infer the stage of NDJ (Meiosis I- MI or Meiosis II-MII). If parental heterozygosity was retained in the trisomic child at the centromeric markers, we considered the case to be of MI origin. If parental heterozygosity was reduced to homozygosity, we interpreted this as an MII origin. MII events with no evidence of recombination were considered to be mitotic errors and were excluded, as described by Lamb et al., 2005 and Oliver et al., 2008.

Another 11 STR markers were used to divide 21q into intervals to monitor the exchange events. After genotyping, the status of each marker was recorded as Non-reduced, reduced, partially informative and uninformative.

\section{Statistical analysis:-}

We performed Chi-square tests of independence and linear regression analysis for our data sets to analyze two aspects of our study- 1) Amount of recombination and 2) position of recombination. We divided families into three groups based on maternal age at the time of the trisomic birth: young ( $\leq 27$ years), middle ( $27-34$ years) and old ( $\geq 34$ years). To examine locations of recombination events by age we performed simple linear regressions of the location (scored as the interval number) on the maternal age. 


\section{Results:- \\ Study population:-}

The population under investigation includes 145 mothers of DS and 130 mothers of normal kids. The parental origin of the segregation error was determined by detecting the contribution of parental alleles to the offspring for multiple markers. We have reported only the cases with maternal meiotic errors. Of these, $117(80.68 \%)$ cases were originated from Meiosis I (MI) and 28 (19.32\%) cases from Meiosis II (MII) (Table 1).

Analysis of recombination profile:-

We made two categories, MI and MII, in order to analyze the recombination profile in these different stages.

\section{Maternal age and MI errors:-}

The data from our study indicated 'absence of recombination' to be higher when compared to single exchange events and multiple exchange events regardless of maternal age. Younger age group mothers were under hammer as this group showed higher frequency of zero exchange/'absence of recombination' and multiple exchange events when compared to other age groups (Table 2). To test its significance, chi square analysis was performed for younger group mothers against middle age group $(\mathrm{P}=0.03)$ and old age group mothers $(\mathrm{P}=0.04)$ which was found to be significant. While the control group showed single exchange event to be higher in frequency indicating single recombination facilitates proper segregation of chromosomes.

Further, placement of these recombination events was observed which pointed 'exchange events' were placed in telomeric region in young age group mothers when compared with middle age and old age group which showed centromeric placed exchanges (Table 3).

\section{Maternal age and MII errors:-}

We initiated the analysis of MII errors by following the same procedures of MI errors. MII with 'absence of recombination' were assumed to be post-zygotic, mitotic errors and were excluded from these analyses. Our data interprets young age mothers had higher frequency of multiple recombination events (35\%) and were at greater risk of giving birth to DS. The frequency of 'multiple recombination events' reduced as the age increases i.e. middle age group (32\%) and old age group (12\%). When this event was compared with young mothers of control group and MI error group, reduction of multiple exchange events was observed. This indicates 'multiple exchange events' as a potential risk factor for young mothers of DS in MII errors (Table 2).

In MII errors, placement of recombination events in all the maternal age groups was observed in centromeric region (Figure 2). Linear regression analysis was performed for recombination events with maternal age as predictor, only young mother age group showed significant association $(\mathrm{p}=0.004)$. Linear regression was performed for each group and the results did not yield any significance (Table 4).

The present investigation indicated mothers of DS in younger age group was associated with recombination errors in causing NDJ leading to DS. The results also indicated similar pattern of recombination events i.e. 'absence of recombination', 'multiple exchange event' and telomeric exchange as susceptible factors indicated in previous studies and plays a role in NDJ. No deviation in recombination event and patterns was observed in south Indian population when compared with previous studies in different geographical area and our data supports the previous studies.

\section{Consanguineous marriage and rate of DS:-}

The overall rate of consanguineous marriage from our investigation was found to be $37.16 \%$ in cases and only $7 \%$ in controls. This indicates the higher proportion consanguineous marriages in this part of world. Young and middle age mothers of DS had higher frequency of consanguineous marriage, whereas older mother group didn't showed higher rate of consanguinity (Table 2). However, no significance $(\mathrm{P}=1.66)$ was observed when chi square was applied for this data.

\section{Discussion:-}

Down syndrome (DS) is one of the most common chromosomal abnormalities causing mental retardation and is of major public health concern. DS is caused due to NDJ of chromosome 21 occurring during maternal meiotic 
division ( $90 \%)$. Errors occur more frequently in the first maternal meiotic division than the second (73\% vs 25\%) (Antonarakis et al., 1992; Yoon et al., 1996; Hassold and Sherman, 2000)

Even after decades of research on DS, causes for NDJ of chromosome 21 are poorly understood. The only well established factors of NDJ of chromosome 21 are maternal age, advanced grandmother age (Malini et al, 2006 and Allen et al, 2009) and altered recombination (Lamb et al, 2005; Sherman et al, 2006; Oliver et al 2009). Altered genetic recombination has been identified as the first molecular correlate of chromosome NDJ in both humans and model organisms. Little evidence has emerged to link maternal age long recognized as the primary risk factor for NDJ with altered recombination, although some studies have provided hints of such a relationship (Lamb et al, 2005, Oliver et al, 2009). Researchers have also tried to look into genome wide recombination dysregulation (Middlebrooks et.al, 2014), recombination associated hotspots (Oliver et.al, 2014); structural variations such as copy number variations (CNVs) for causing aneuploidy (Suresh et. al, 2015, in press) but maternal age, advanced grandmother age and altered recombination emerge as important ones. The present investigation was taken up to provide further insights into the established factors, we present a case-control study on NDJ of DS based on recombination errors. The study was specific to South Indian population and is of particular importance as consanguineous marriages and inbreeding are high in this region.

In the present investigation, our focus was on meiosis occurring in the advancing oocyte. Several meiotic proteins that function to promote proper chromosome segregation have been shown to degrade with increasing age (Baker et.al, 2004, Steuerwald et.al, 2001). This degradation is assumed to lead to increased frequency of NDJ; thus, more maternal-age related risk factors for NDJ exist among older women compared to younger women. Our analysis compared the predisposing recombination patterns among the oocytes with nondisjoined events by maternal age.

As recombination plays a major role in meiosis process to facilitate proper alignment of homologous chromosomes, any variations in the recombination will hamper the above process. Hence, our analysis begins with the identification of amount of recombination specific to chromosome 21 in cases and control mothers.

We started our present investigation by identifying the stage of origin for NDJ in DS using specific STR markers. Once the origin i.e. either meiosis I error (MI) or meiosis II error (MII) were determined, we calculated the amount of recombination in each meiotic outcome group stratified by maternal age (young, middle and old age). MI outcome group showed two major findings. The first finding i.e. absence of recombination to be significantly higher (64\%) (Table 2) when compared to single or multiple exchanges. This reveals in the absence of the exchange event, homologous chromosomes are at risk for malsegregation during MI and the second finding showed presence of telomere exchange in young mothers with DS. In contrast to the younger mothers, the DS mothers with older age group showed lesser percent (51\%) absence of recombination and the crossover took place in the centromeric region (Figure 2) and showed significant linear shift $(\mathrm{p}=0.004)$. our data for MI errors hypothesizes that distally placed chiasmata appear to impose susceptibility for chromosomal malsegregation irrespective of age and Supports same hypothesis made among model organisms (Zetka and Ross, 1995; Koehler et al., 1996a; Ross et al., 1996). Distally placed single chiasma links the homologues less efficiently due to recruitment of a minimum amount of sister chromatid cohesion complex which prevents the bi orientation and subsequent separation of homologues on the meiotic spindle (Hawley et al., 1994; Koehler et al., 1996b; Orr-Weaver, 1996). Alternatively, the integrity of the chiasma may be compromised when a minimal amount of cohesin remains to hold homologues together. Thus, bivalents may act as a pair of functional univalents during MI, as has been observed in human oocytes (Angell 1995, Angell et.al, 1994).

In meiosis II (MII) errors multiple recombination events were higher $(35 \%)$ in young mothers, while the middle age women $(32 \%)$ and old age women $(12 \%)$ with DS kids showed decrease frequency in these events (Table 2). These data supports 'multiple recombinations' as one of the risk factors in causing NDJ by Oliver et.al, (2012). The location of these exchange events were placed more in centromeric and pericentomeric region in all the age group. This indicated possible shift of crossover event from telomeric region to centromeric region in younger mother group and was found statistically significant (Figure 2).

The control samples in our study showed less multiple recombination events, where as absence of recombination was lesser than that of the case groups. The recombination events took place more in centromeric region signifying crossover events in centromeric region for normal disjoining of chromosomes. 
The present investigation displays the effect of recombination errors and its placement in younger mothers as one of major factors in causing NDJ. In conclusion, our examination reveals recombination errors, recombination pattern and 'young' maternal age plays a major role in chromosomal NDJ and can act as predisposing factors for NDJ of chromosome 21 leading to Down syndrome.

\section{Conflict of interest:-}

The authors declare no conflict of interests.

\section{Acknowledgement:-}

The authors thank all the subjects participated in the study. We also thank all our lab mates, Genetics and Genomics lab for their support.

Funding Agencies:-

We thank the funding agencies, University Grants Commission UGC-BSR (DV5/323/RFSMS/2006-07) and UGCBSR one time grant.

Figure legends:-

\section{PHYSICALLOCATIONS (bp)}

13667220

17137386

23735731

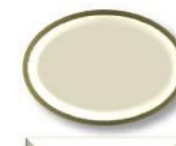

STR MARKERS

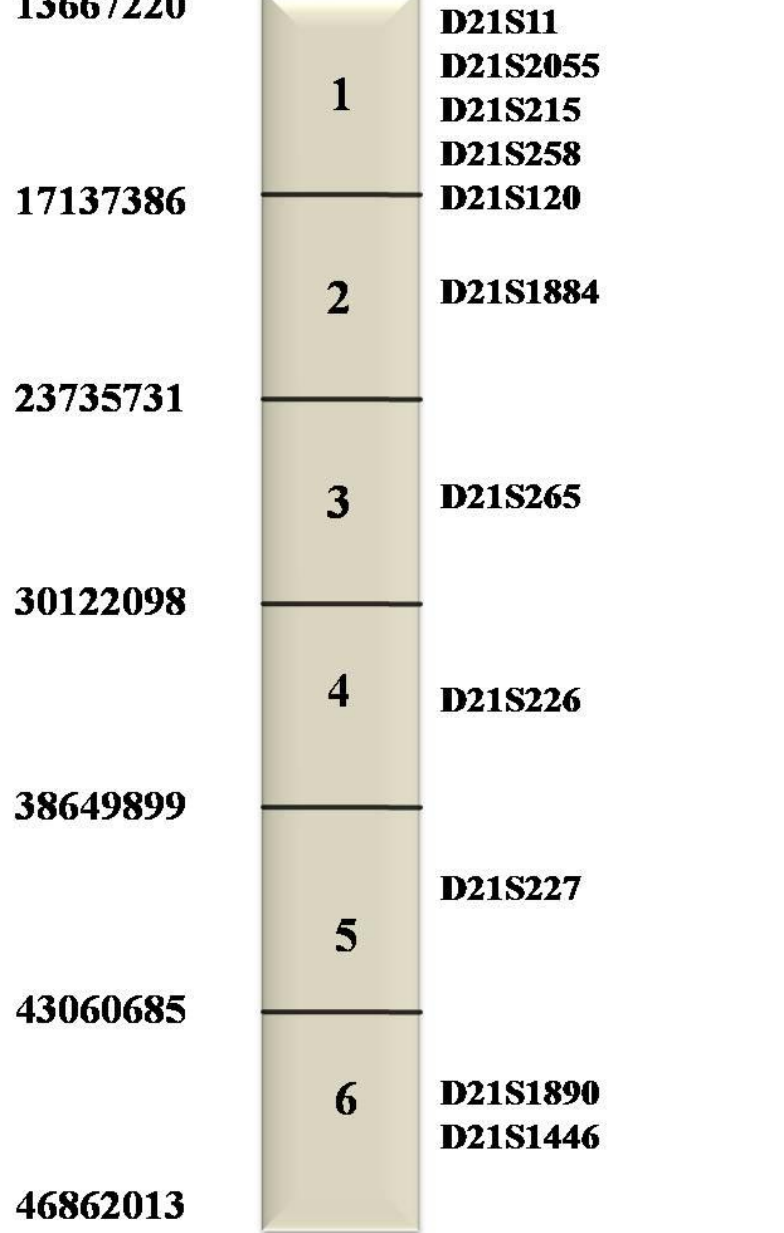

Figure 1: STR markers used determine parental origin, stage of nondisjunction and recombination profile with their physical location on chromosome 21. 


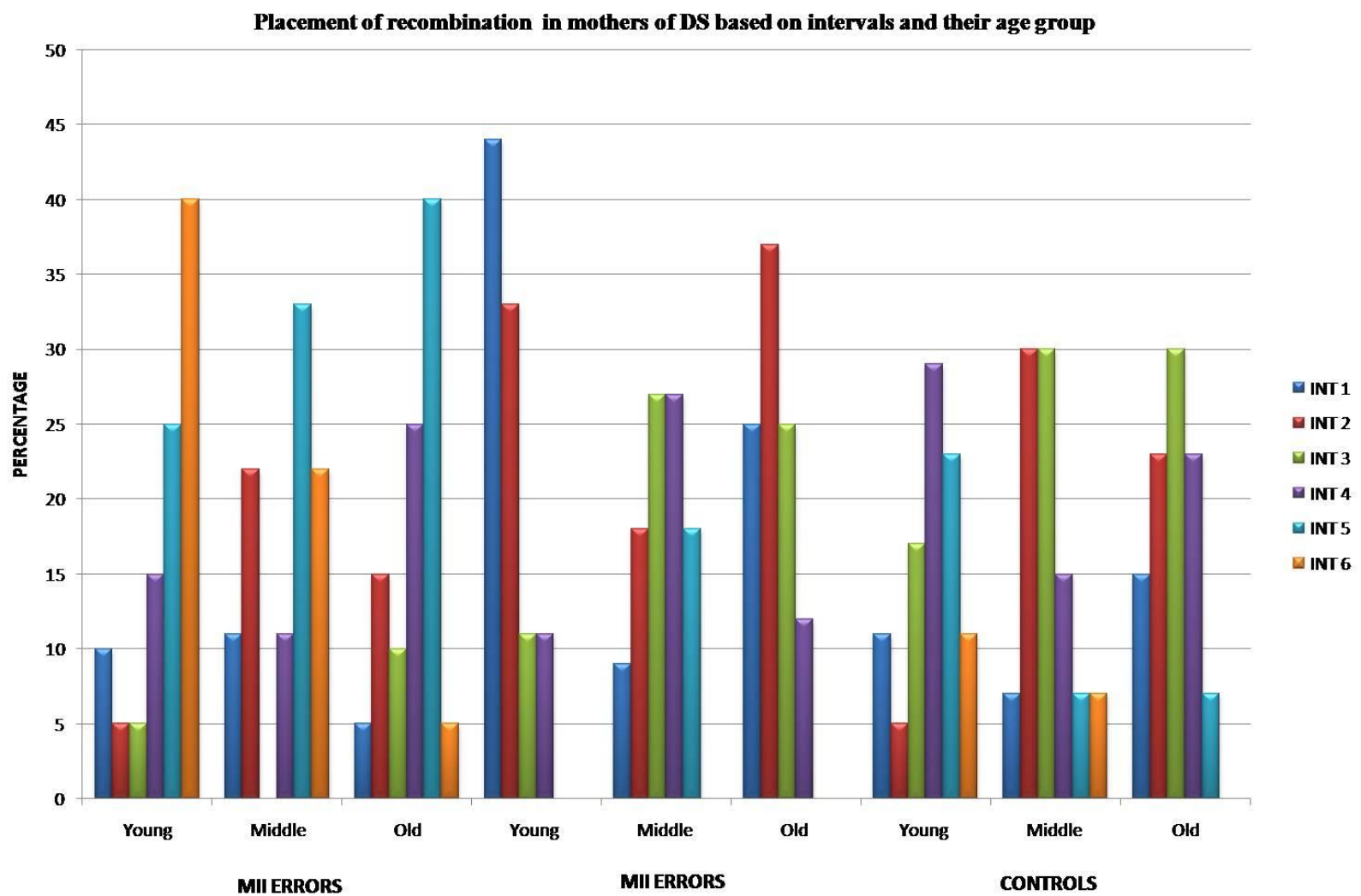

Figure 2: Maternal age stratified crossover events specific to chromosome 21 in meiosis I and meiosis II stages and their comparison with case and control samples.

Tables:-

Supplementary Table 1: STR markers used in the study with their primer sequence information.

\begin{tabular}{|c|c|}
\hline STR MARKERS & PRIMER SEQUENCE \\
\hline D21S11 & $\begin{array}{l}\text { GTGAGTCAATTCCCCAAG (F) } \\
\text { GTTGTATTAGTCAATGTTCTCC (R) }\end{array}$ \\
\hline D21S2055 & $\begin{array}{l}\text { AACAGAAACCAATAGGCTATCTTATC (F) } \\
\text { TACAGTAAATCACTTGGTAGGAGA }(\mathrm{R})\end{array}$ \\
\hline D21S171 & $\begin{array}{l}\text { GATCAAGTTAAGAGGAGGCT (F) } \\
\text { GTGGGCTGCTGCTATCTTTG (R) }\end{array}$ \\
\hline D21S215 & $\begin{array}{l}\text { GCTGACGTGACAGTTGTGAG (F) } \\
\text { TCTAAAACAGTGTGTCTAGC (R) }\end{array}$ \\
\hline D21S120 & $\begin{array}{l}\text { GTGTGTCTGCCATTTCTGGGTGTAG (F) } \\
\text { GATCCTGGGACAAAGTAGTCTCTAA (R) }\end{array}$ \\
\hline D21S1414 & $\begin{array}{l}\text { GGCACCCAGTAAAAAATTACT (F) } \\
\text { CTGTCTGTCTGTCTGTCTATC (R) }\end{array}$ \\
\hline D21S1446 & $\begin{array}{l}\text { ACCATCTTACTGGTTTATGTAT (F) } \\
\text { GTCCCAAAGGACCTGCTC }(\mathrm{R})\end{array}$ \\
\hline D21S1890 & $\begin{array}{l}\text { GGTCTGACCACAGATTTCC (F) } \\
\text { AAAAACACTCTGAACGATTAAGG (R) }\end{array}$ \\
\hline D21S267 & $\begin{array}{l}\text { GGGGATTATTTATGTAGAAAATGAGA(F) } \\
\text { GGTGACAGACCCTGTCTCTAAAA(R) }\end{array}$ \\
\hline D21S226 & $\begin{array}{l}\text { AGCTAAATGTCTGTAGTTATT(F) } \\
\text { GGAAACCACTCTAAGACATA(R) }\end{array}$ \\
\hline D21S265 & $\begin{array}{l}\text { GGGTAAGAAGGTGCTTAATGCTC(F) } \\
\text { TGAATATGGGTTCTGGATGTAGTG(R) }\end{array}$ \\
\hline D21S1884 & $\begin{array}{l}\text { TGATGCAAAAAATTATTGATAAC(F) } \\
\text { GATGTGAATACAGGCAGATG(R) }\end{array}$ \\
\hline
\end{tabular}


Table 1: Number of individuals under the study in maternal age stratified meiotic stage errors in DS mothers

\begin{tabular}{|c|c|c|c|c|}
\hline Sl.No & Age group & Maternal meiosis I & Maternal meiosis II & Total \\
\hline $\mathbf{1}$ & $\begin{array}{c}\text { Young age } \\
(<26 \text { years })\end{array}$ & $55(47 \%)$ & $9(32.14 \%)$ & $64(44.13 \%)$ \\
\hline $\mathbf{2}$ & $\begin{array}{c}\text { Middle age } \\
(27-33 \text { years })\end{array}$ & $22(18.80 \%)$ & $11(39.28 \%)$ & $33(22.75 \%)$ \\
\hline $\mathbf{3}$ & $\begin{array}{c}\text { Old age } \\
(\geq 34 \text { years })\end{array}$ & $40(34.20)$ & $8(28.57 \%)$ & $48(33.10 \%)$ \\
\hline & Total & $117(80.68 \%)$ & $28(19.32 \%)$ & 145 \\
\hline
\end{tabular}

Table 2: Frequency distribution of observed recombination events for maternally derived nondisjunction groups among different maternal age groups

\begin{tabular}{|c|c|c|c|c|c|c|}
\hline Recombinant frequency observed \\
\hline Meiotic outcome group & Maternal Age Quartile & Sample Size & $\begin{array}{c}\text { Consanguineous } \\
\text { marriages (\%) }\end{array}$ & $\mathbf{0}$ & $\mathbf{1}$ & $\geq \mathbf{2}$ \\
\hline & Young $(<26$ years) & 55 & $21(41.17)$ & 0.64 & 0.2 & 0.16 \\
\hline MI & Middle (27-33 years) & 22 & $08(36.36)$ & 0.6 & 0.3 & 0.1 \\
\hline & Old $(\geq 34$ years) & 40 & $13(32.5)$ & 0.51 & 0.42 & 0.07 \\
\hline & Young (<26 years) & 9 & $04(44.44)$ & - & 0.65 & 0.35 \\
\hline MII & Middle $(27-33$ years) & 11 & $06(54.54)$ & - & 0.68 & 0.32 \\
\hline & Old $(\geq 34$ years) & 8 & $03(37.5)$ & - & 0.88 & 0.12 \\
\hline & Young $(<26$ years) & 57 & $06(10.52)$ & 0.50 & 0.46 & 0.03 \\
\hline CONTROLS & Middle $(27-33$ years) & 42 & $03(7.14)$ & 0.59 & 0.36 & 0.04 \\
\hline & Old $(\geq 34$ years) & 31 & - & 0.58 & 0.35 & 0.06 \\
\hline
\end{tabular}

Table 3: Distribution of crossover for each meiotic outcome group on chromosome 21 based on intervals stratified by maternal age

\begin{tabular}{|c|c|c|c|c|c|c|c|}
\hline Meiotic outcome group & Maternal age quartile & \multicolumn{7}{|c|}{ Interval location of inferred exchange } & \\
\hline & & $\mathbf{1}$ & $\mathbf{2}$ & $\mathbf{3}$ & $\mathbf{4}$ & $\mathbf{5}$ & $\mathbf{6}$ \\
\hline & Young(<26 years) & 0.1 & 0.05 & 0.05 & 0.15 & 0.25 & 0.4 \\
\hline & Middle(27-33 Years) & 0.11 & 0.22 & 0 & 0.11 & 0.33 & 0.22 \\
\hline & Old( $\geq 34$ years) & 0.05 & 0.15 & 0.1 & 0.25 & 0.4 & 0.05 \\
\hline & Young $(<26$ years) & 0.44 & 0.33 & 0.11 & 0.11 & 0 & 0 \\
\hline & Middle (27-33 Years) & 0.09 & 0.18 & 0.27 & 0.27 & 0.18 & 0 \\
\hline MII & Old $(\geq 34$ years) & 0.25 & 0.37 & 0.25 & 0.12 & 0 & 0 \\
\hline & Young (<26 years) & 0.11 & 0.05 & 0.17 & 0.29 & 0.23 & 0.11 \\
\hline CONTROL & Middle $(27-33$ Years) & 0.07 & 0.3 & 0.3 & 0.15 & 0.07 & 0.07 \\
\hline & Old $(\geq 34$ years) & 0.15 & 0.23 & 0.3 & 0.23 & 0.07 & 0 \\
\hline
\end{tabular}

Table 4: Linear regression analysis performed for maternal age groups and their P values.

\begin{tabular}{|l|l|l|l|l|l|}
\hline & Young vs Middle & Young vs Old & Control vs Young & Control vs Middle & Control vs old \\
\hline Standard Error & 0.4043 & 0.3982 & 2.3209 & 1.9127 & 0.8706 \\
\hline t Stat & 14.7215 & 13.4394 & 1.1732 & 2.3930 & 5.9722 \\
\hline P-value & 0.0006 & 0.0008 & 0.3253 & 0.0964 & 0.0093 \\
\hline Lower 95\% & 4.6660 & 4.0849 & -4.6631 & -1.5099 & 2.4290 \\
\hline Upper 95\% & 7.2397 & 6.6198 & 10.1094 & 10.6648 & 7.9709 \\
\hline
\end{tabular}




\section{References:-}

1. Angell, R. (1997): First-meiotic-division nondisjunction in human oocytes. The American Journal of Human Genetics., 61(1), 23-32.

2. Angell, R. R., Xian, J., Keith, J., Ledger, W., \& Baird, D. T. (1994): First meiotic division abnormalities in human oocytes: mechanism of trisomy formation. Cytogenetic and Genome Research., 65(3), 194-202.

3. Allen, E. G., Freeman, S. B., Druschel, C., Hobbs, C. A., O’Leary, L. A., Romitti, P. A., ... \& Sherman, S. L. (2009): Maternal age and risk for trisomy 21 assessed by the origin of chromosome nondisjunction: a report from the Atlanta and National Down Syndrome Projects. Human Genetics., 125(1), 41-52.

4. Antonarakis, S. E. (1991): Parental origin of the extra chromosome in trisomy 21 as indicated by analysis of DNA polymorphisms. New England Journal of Medicine., 324(13), 872-876.

5. Baker, D. J., Jeganathan, K. B., Cameron, J. D., Thompson, M., Juneja, S., Kopecka, A., ... \& van Deursen, J. M. (2004): BubR1 insufficiency causes early onset of aging-associated phenotypes and infertility in mice. Nature Genetics., 36(7), 744-749.

6. Cheung, V. G., Burdick, J. T., Hirschmann, D., \& Morley, M. (2007): Polymorphic variation in human meiotic recombination. The American Journal of Human Genetics., 80(3), 526-530.

7. Freeman, S. B., Allen, E. G., Oxford-Wright, C. L., Tinker, S. W., Druschel, C., Hobbs, C. A \& Sherman, S. L. (2007): The National Down syndrome project: design and implementation. Public Health Reports., 62-72.

8. Ghosh, S., Feingold, E., \& Dey, S. K. (2009): Etiology of Down syndrome: Evidence for consistent association among altered meiotic recombination, nondisjunction, and maternal age across populations. American Journal of Medical Genetics Part A., 149(7), 1415-1420.

9. Hawley, R. S., Frazier, J. A., \& Rasooly, R. (1994): Separation anxiety: the etiology of nondisjunction in flies and people. Human molecular Genetics., 3(9), 1521-1528.

10. Hassold, T., Merrill, M., Adkins, K., Freeman, S., \& Sherman, S. (1995): Recombination and maternal age-dependent nondisjunction: molecular studies of trisomy 16. American journal of human Genetics., 57(4), 867.

11. Koehler, K. E., Boulton, C. L., Collins, H. E., French, R. L., Herman, K. C., Lacefield, S. M., ... \& Hawley, R. S. (1996): Spontaneous X chromosome MI and MII nondisjunction events in Drosophila melanogaster oocytes have different recombinational histories. Nature Genetics., 14(4), 406-414.

12. Koehler, K. E., Hawley, R. S., Sherman, S., \& Hassold, T. (1996): Recombination and nondisjunction in humans and flies. Human Molecular Genetics., 5(Supplement 1), 1495-1504.

13. Lamb, N. E., Yu, K., Shaffer, J., Feingold, E., \& Sherman, S. L. (2005): Association between maternal age and meiotic recombination for trisomy 21. The American Journal of Human Genetics., 76(1), 91-99.

14. Malini, S. S., \& Ramachandra, N. B. (2006): Influence of advanced age of maternal grandmothers on Down syndrome. BMC Medical Genetics., 7(1), 4.

15. MacDonald, M., Hassold, T., Harvey, J., Wang, L. H., Morton, N. E., \& Jacobs, P. (1994): The origin of 47, XXY and 47, XXX aneuploidy: heterogeneous mechanisms and role of aberrant recombination. Human molecular Genetics., 3(8), 1365-1371.

16. Oliver, T. R., Feingold, E., Yu, K., Cheung, V., Tinker, S., Yadav-Shah, M \& Sherman, S. L. (2008): New insights into human nondisjunction of chromosome 21 in oocytes. PLoS Genetics., 4(3), e1000033.

17. Orr-Weaver, T. (1996): Meiotic nondisjunction does the two-step. Nature Genetics., 14(4), 374-376.

18. Ross, L. O., Maxfield, R., \& Dawson, D. (1996): Exchanges are not equally able to enhance meiotic chromosome segregation in yeast. Proceedings of the National Academy of Sciences., 93(10), 4979-4983.

19. Steuerwald, N., Cohen, J., Herrera, R. J., Sandalinas, M., \& Brenner, C. A. (2001): Association between spindle assembly checkpoint expression and maternal age in human oocytes. Molecular Human Reproduction., 7(1), 49-55.

20. Sherman, S. L., Lamb, N. E., \& Feingold, E. (2006): Relationship of recombination patterns and maternal age among non-disjoined chromosomes 21. Biochemical Society Transactions., 34(4), 578-580.

21. Vraneković, J., Božović, I. B., Grubić, Z., Wagner, J., Pavlinić, D., Dahoun, S. \& Brajenović-Milić, B. (2012): Down syndrome: Parental origin, recombination, and maternal age. Genetic Testing and Molecular Biomarkers., 16(1), 7073.

22. Wolstenholme, J., \& Angell, R. R. (2000): Maternal age and trisomy-a unifying mechanism of formation. Chromosoma., 109(7), 435-438.

23. Yoon, P. W., Freeman, S. B., Sherman, S. L., Taft, L. F., Gu, Y., Pettay, D \& Hassold, T. J. (1996): Advanced maternal age and the risk of Down syndrome characterized by the meiotic stage of chromosomal error: a populationbased study. American journal of Human Genetics., 58(3), 628.

24. Zetka, M. C., \& Rose, A. M. (1995): Mutant rec-1 eliminates the meiotic pattern of crossing over in Caenorhabditis Elegans. Genetics., 141(4), 1339-1349.

25. http://www.who.int/genomics/public/geneticdiseases/en/index1.html 